\title{
openheart B-type natriuretic peptide-guided therapy for perioperative medicine?
}

\author{
Charles Shang
}

To cite: Shang C. B-type natriuretic peptide-guided therapy for perioperative medicine?. Open Heart 2014:1:e000105. doi:10.1136/openhrt-2014000105

Received 28 February 2014 Revised 22 May 2014 Accepted 15 July 2014
CrossMark

Department of Medicine, Baylor College of Medicine, Houston, Texas, USA

Correspondence to Dr Charles Shang; cshang9@gmail.com

\section{ABSTRACT}

The recent guideline from the European Society of Cardiology and European Society of Anesthesiology recommended the use of B-type natriuretic peptide (BNP) as preoperative testing for high-risk cardiac patients undergoing non-cardiac surgery. In this article, the potential benefits, risks and details for implementing BNP testing in perioperative medicine are discussed. Review of four related lines of research including the use of BNP test for preoperative prognosis, BNP test for screening asymptomatic heart failure, BNP as prognostic test in asymptomatic, nonheart failure patients and using BNP for detecting silent myocardial ischaemia showed converging cut-off levels of BNP for risk stratification. BNP has better OR and relative risk in comparison with Revised Cardiac Risk Index (RCRI) in predicting perioperative cardiac risk. BNP-guided therapy can be low risk based on current evidence on non-surgical patients, including treating asymptomatic patients without heart failure to prevent cardiovascular complications. At present, there is lack of direct evidence supporting perioperative BNP testing. Further research with randomised controlled trials is needed to confirm the benefit of BNP-guided management. Preoperative BNP testing may be considered in patients with RCRI above 0 undergoing intermediate or high-risk surgery. BNP-guided therapy is likely a beneficial addition to perioperative medicine. Its combination with $\beta$-blocker titration, RCRI and perioperative cardiovascular monitoring can be a major advance in reducing cardiac risk resulting in a dynamic, individualised optimisation process.

\section{INTRODUCTION}

The recent guideline from European Society of Cardiology (ESC) and European Society of Anesthesiology (ESA) recommended using B-type natriuretic peptide (BNP) or amino terminal-proBNP (NT-proBNP) for independent perioperative prognosis in high-risk cardiac patients undergoing non-cardiac surgery. ${ }^{1}$ Both BNP and NT-proBNP are derived from proBNP which is synthesised by myocytes and fibroblasts in response to ventricular filling pressures and wall stress. BNP has sympathoinhibitory effects and reduces secretion of renin, angiotensin and

\section{KEY MESSAGES}

What is already known about this subject?

- BNP is superior to RCRI in predicting perioperative cardiac risk. BNP guided therapy is beneficial and low risk for heart failure patients under age 75 .

What does this study add?

- Integrating BNP guided therapy with current guidelines on perioperative medical management.

How might this impact on clinical practice?

- Perioperative BNP guided therapy can lead to a dynamic, individualized approach of reducing perioperative cardiac risk. Its synergistic combination with beta-blockade can enhance the benefit and reduce the harm of perioperative beta-blockade. At present, the benefit of perioperative BNP guided therapy still await confirmation of further research.

aldosterone. BNP also decreases blood pressure, increases diuresis and vasodilation. BNP is degraded by endopeptidases with a half-life of 5-10 min. ${ }^{2}$ NT-proBNP was the best among 30 biomarkers for improving cardiovascular risk estimation in patients who had no history of heart disease in two recent large prospective cohort studies. ${ }^{3}$ A similar conclusion was reached on BNP in a study using 10 biomarkers. ${ }^{4} \quad$ Preoperative BNP (including NT-proBNP) level has been confirmed by two meta-analyses as a powerful independent predictor of short-term cardiovascular complications within 30-43 days of non-cardiac surgery with OR above $19 .{ }^{5}{ }^{6} \mathrm{~A}$ similar meta-analysis on the use of preoperative BNP in vascular surgical patients also showed high OR above 17 in predicting major cardiac complications. ${ }^{7}$

A recent cohort study concluded that preoperative BNP was the only clinically useful predictor of postoperative cardiac complications. ${ }^{8}$ BNP is a strong prognostic indicator of death and cardiac events for both asymptomatic patients ${ }^{9-12}$ and patients with heart failure. ${ }^{13}$ In heart failure patients, each $100 \mathrm{pg} / \mathrm{mL}$ increase was associated with a 
$35 \%$ increase in the relative risk of death. ${ }^{13}$ In patients without heart failure, each increment of 1 SD in $\log$ BNP levels was associated with a $27 \%$ increase in the relative risk of death, a $28 \%$ increase in the relative risk of a first cardiovascular event, a $77 \%$ increase in the relative risk of heart failure after adjustment for cardiovascular risk factors. ${ }^{10}$ The results of large prospective cohort studies suggest that slight elevations of BNP may reflect early stages of pathological processes that precede the development of apparent cardiac manifestations. BNP measurement may provide a very early warning signal for future cardiovascular disease in persons without symptoms. ${ }^{14}$ It is likely that BNP elevation identifies patients with impaired cardiac function or a significant ischaemic risk who may not withstand the haemodynamic and proinflammatory stresses of general anaesthesia and major surgery. ${ }^{6}$

A literature search on Scopus database (the largest abstract and citation database of peer-reviewed research literature which encompasses MEDLINE, EMBASE and Cochrane databases) using '(BNP OR natriuretic) AND (preoperative OR perioperative)' yielded 811 articles from 1995 to present. When 'randomised' was added as keyword to limit the results, the number of publications was reduced to 110. A review of the title and abstract of the articles showed that there has been no prospective randomised controlled trial using BNP-guided management in perioperative medicine despite related research suggesting the potential benefit of perioperative BNP testing. How BNP testing should be integrated into current perioperative medicine guidelines has not been established. In this article, the benefits, risks and a detailed algorithm for application of BNP testing in perioperative medicine are discussed based on current evidence which includes not only the perioperative observational studies, but also the related studies on the use of BNP testing in heart failure, silent ischaemia and asymptomatic patients.

\section{Criteria for BNP testing in preoperative patients}

BNP was shown to have sensitivity of $87 \%$ and specificity of $87 \%$ in predicting cardiovascular events for noncardiac vascular surgery in a prospective derivation study. ${ }^{15}$ The commonly used Revised Cardiac Risk Index (RCRI) which includes ischaemic heart disease, heart failure, diabetes requiring insulin, cerebrovascular disease, renal insufficiency with creatinine above $2 \mathrm{mg} / \mathrm{dL}$ as 5 independent predictors with a score from 0 to 5 based on the number of risk factors, only showed sensitivity of $64 \%$ and specificity of $70 \%$ in predicting cardiovascular events in a recent meta-analysis. ${ }^{16}$ In several head-to-head comparison study of BNP or NT-proBNP versus RCRI, preoperative BNP has shown better $\mathrm{OR}^{17}$ and relative risk ${ }^{18}$ than RCRI in predicting perioperative cardiovascular complications. BNP remains independently predictive after correction for RCRI in several studies. ${ }^{19} 20$ RCRI and BNP are independent perioperative predictors of cardiac dysfunction and mortality. ${ }^{18}$ Combining BNP, RCRI and $\mathrm{C}$ reactive protein (CRP) predicted better relative risk than any one alone for preoperative cardiac risk stratification. ${ }^{18}$ Since the sensitivity and specificity of BNP have been shown to be better than those of RCRI, it would be more appropriate to use a lower threshold of RCRI to initiate BNP measurement than using the conventional high-risk threshold of RCRI above 1. Some cohort studies have shown that BNP provides prognostic information of mortality and first major cardiovascular events beyond traditional risk factors. NT-proBNP has been shown to be a stronger risk biomarker for cardiovascular disease and death than CRP in patients without heart failure. $^{321}$ On the other hand, a recent study suggests that NT-proBNP was not predictive of death or cardiovascular events in patients without traditional clinical cardiovascular risk factors and echocardiographic structural cardiac abnormalities. ${ }^{22}$ BNP measurement is unlikely to be cost-effective in patients with RCRI of 0 in light of the low risk in this population. Therefore, RCRI above 0 is a reasonable threshold for using BNP testing in patients who require intermediate or high-risk surgeries. This is consistent with the recent proposal of using BNP as a screening test to target specific subpopulations with borderline or single traditional risk factor for coronary artery disease. ${ }^{23}$ BNP may also be considered for preoperative evaluation in patients undergoing intermediate or high-risk surgeries with heart disease, history of elevated BNP or abnormal ECG. The current guidelines recommend preoperative ECG for all vascular surgical patients and patients undergoing intermediate risk surgery with RCRI $>0,{ }^{1}$ or peripheral vascular disease. ${ }^{24}$ Since the cost of BNP test $(\sim \mathrm{US} \$ 20)^{6}$ is comparable to the cost of performing and interpreting ECG while the evidence supporting BNP for preoperative risk stratification is stronger than that of ECG, ${ }^{25} 26$ BNP testing should be considered in patients who meet the criteria of preoperative ECG which is very similar to the criteria of RCRI $>0$ for patients undergoing intermediate or high-risk surgeries.

\section{The optimal cut-off BNP level for distinguishing low-risk versus high-cardiac risk}

Two meta-analyses published in 2009 could not define the optimal cut-off value for BNP and NT-proBNP due to wide variation between studies. ${ }^{5}{ }^{6} \mathrm{~A}$ more recent meta-analysis suggested that the screening BNP cut point was $30 \mathrm{pg} / \mathrm{mL}$ for predicting cardiac death and non-fatal myocardial infarction within 30 days of noncardiac vascular surgery with $95 \%$ sensitivity and $44 \%$ specificity. ${ }^{27}$ This level is quite consistent with other studies using BNP to predict cardiovascular events in asymptomatic patients which is similar to the task of preoperative cardiac evaluation of asymptomatic surgical patients. Excess risk was apparent at BNP levels well below current thresholds used to diagnose acute heart failure: In a large cohort study on asymptomatic patients who started without diagnosis of heart failure, BNP 
values above $20.0 \mathrm{pg} / \mathrm{mL}$ for men and $23.3 \mathrm{pg} / \mathrm{mL}$ for women were associated with multivariable-adjusted HRs of 1.62 for death, 1.76 for a first major cardiovascular event and 3.07 for heart failure during a mean follow-up of 5.2 years. ${ }^{10}$ Similar BNP threshold of increasing cardiac risk were found in several other large cohort studies. ${ }^{28}{ }^{29}$ A review suggested that the optimal cut points for excluding heart failure in asymptomatic patients are BNP $<20 \mathrm{pg} / \mathrm{mL}$ and NT-proBNP $<125 \mathrm{pg} /$ $\mathrm{mL}^{30}$ which has been supported by the recent PROBE-HF study. ${ }^{31}{ }^{32}$ Furthermore, studies have shown that $\mathrm{BNP}>20 \mathrm{pg} / \mathrm{mL}$ produced $87 \%$ sensitivity, $37 \%$ specificity and a negative predictive accuracy of $90 \%$ at detecting silent ischaemic heart disease in patients with diabetes. ${ }^{33}$ Heart failure and ischaemic heart disease are well-established risk factors of perioperative cardiac complications and are part of the RCRI. ${ }^{24}$ Based on converging results from several lines of related research discussed above, BNP cut-off value is likely around $20-30 \mathrm{pg} / \mathrm{mL}$ and NT-proBNP cut-off value is likely around $125 \mathrm{pg} / \mathrm{mL}^{31}{ }^{32}$ for preoperative screening purposes in moderate to high-risk patients. The convergence of results from several related lines of research further suggests that the cut-off value of BNP for vascular surgerical patients may be similar to non-vascular surgical patients. Some study also suggests normal NT-Pro-BNP levels should be stratified by age and gender to improve the negative predictive values of cardiovascular diseases. ${ }^{34}$

Another controversy is the utility of BNP testing in patients with renal impairment. A recent cohort study involving 356 patients undergoing non-cardiac vascular surgery showed that the prognostic value of NT-pro-BNP decreased with the patients' glomerular filtration rate. In this study, the NT-pro-BNP had no prognostic value in patients with GFR $<30 \mathrm{~mL} / \mathrm{min} / 1.73 \mathrm{~m}^{2}{ }^{35}$ However, there were only 18 patients with GFR $<30$ and their event rate was more than $40 \%$ in this study. On the other hand, many studies have shown that NT-pro-BNP has prognostic value for heart failure patients with renal impairment, ${ }^{36}$ including patients on dialysis. ${ }^{37}$ The conclusion from a recent review suggests that the cut-off points of NT-proBNP do not require adjustment with renal impairment. ${ }^{36}$

\section{BNP-guided therapy in perioperative medicine}

While preoperative revascularisation is costly and has not shown significant benefit in stable patients, ${ }^{1} 2438$ BNP-guided therapy has not been prospectively tested in perioperative medicine but appears to be safe ${ }^{39}$ and costeffective in stable heart failure patients. ${ }^{40-42}$ In inpatient and outpatient studies, patients with the largest relative reductions in BNP levels had the most favourable outcomes, whereas patients with the greatest percentage increase in BNP were observed to have the highest cardiac event rates. ${ }^{2}$ A meta-analysis of randomised controlled trials has shown that BNP-guided therapy reduced the relative risk of all cause mortality by $48 \%$ in heart failure patients under age $75 .^{39} 43$ A randomised controlled trial on asymptomatic patients with risk factors but without heart failure also showed relative reduction of major cardiovascular events over $40 \%$ using BNP-guided therapy as prevention. ${ }^{44}$ It is encouraging that BNP-guided therapy led to relative reduction of major cardiovascular events over $40 \%$ both as prevention of heart failure in randomisedcontrolled trial (RCT) and as direct treatment of heart failure in meta-analysis. None of the meta-analysis or RCTs has detected any significant increase in adverse events from BNP-guided therapy in comparison with standard care. ${ }^{39}$ It is still controversial regarding the application of BNP-guided therapy in elderly above age 75 . A recent RCT showed that BNP-guided therapy can be more effective in elderly over age 75 when the therapy was gradually titrated with small increment of dosing adjustment and close follow-up. ${ }^{45}$ A recent prospective study involving 1918 stable asymptomatic patients suggested that simple medication switch involving mostly one clinic visit can lead to BNP reduction associated with $13 \%$ potential relative reduction in mortality. ${ }^{46}$ This study also suggested that further up titration beyond one clinic visit is much less cost-effective in asymptomatic patients. ${ }^{46}$ Therefore, a cost-effective perioperative BNP-guided therapy in stable patients should aim to limit preoperative medication titration to no more than one extra clinic visits beyond the current standard of care. It has been proposed to start BNP-guided therapy for preoperative medical optimisation 4-5 weeks before surgery. ${ }^{42}$ Elevated BNP is common in patients with renal impairment due to poorly controlled hypervolemia, heart failure and/or other reasons. Medication adjustment such as titrating diuresis, nitrate and hydrazine may be ineffective for many of them. This is unlikely to be a major problem for using the algorithm which recommends the preoperative medication titration to be mostly limited to one round as long as BNP is not trending up significantly.

Based on a recent review of BNP-guided therapy trials, ${ }^{47}$ the perioperative medicine guideline ${ }^{1}$ and the other evidence listed above, the following algorithm of BNP-guided therapy may be considered for perioperative medicine (figure 1).

If BNP is less than the optimal cut-off value, proceed with the current American College of Cardiology/ American Heart Association (ACC/AHA) ${ }^{24}$ or ESC/ESA guideline with no more BNP tests unless patients show new signs of cardiovascular stress (eg, angina, tachycardia, dyspnoea, ECG change). If BNP is above the optimal cut-off value, use serial BNP-guided therapy with the goal of reducing BNP below the cut-off level by substituting calcium channel blockers, increasing reninangiotensin-aldosterone blockade, diuresis, fluid restriction and/or nitrate +/- hydralazine. Avoid hypotension (eg, keep systolic blood pressure $>100 \mathrm{~mm} \mathrm{Hg})^{1}$ or worsening renal function. Since angiotensin blockade and diuresis are associated with higher risk of intraoperative 


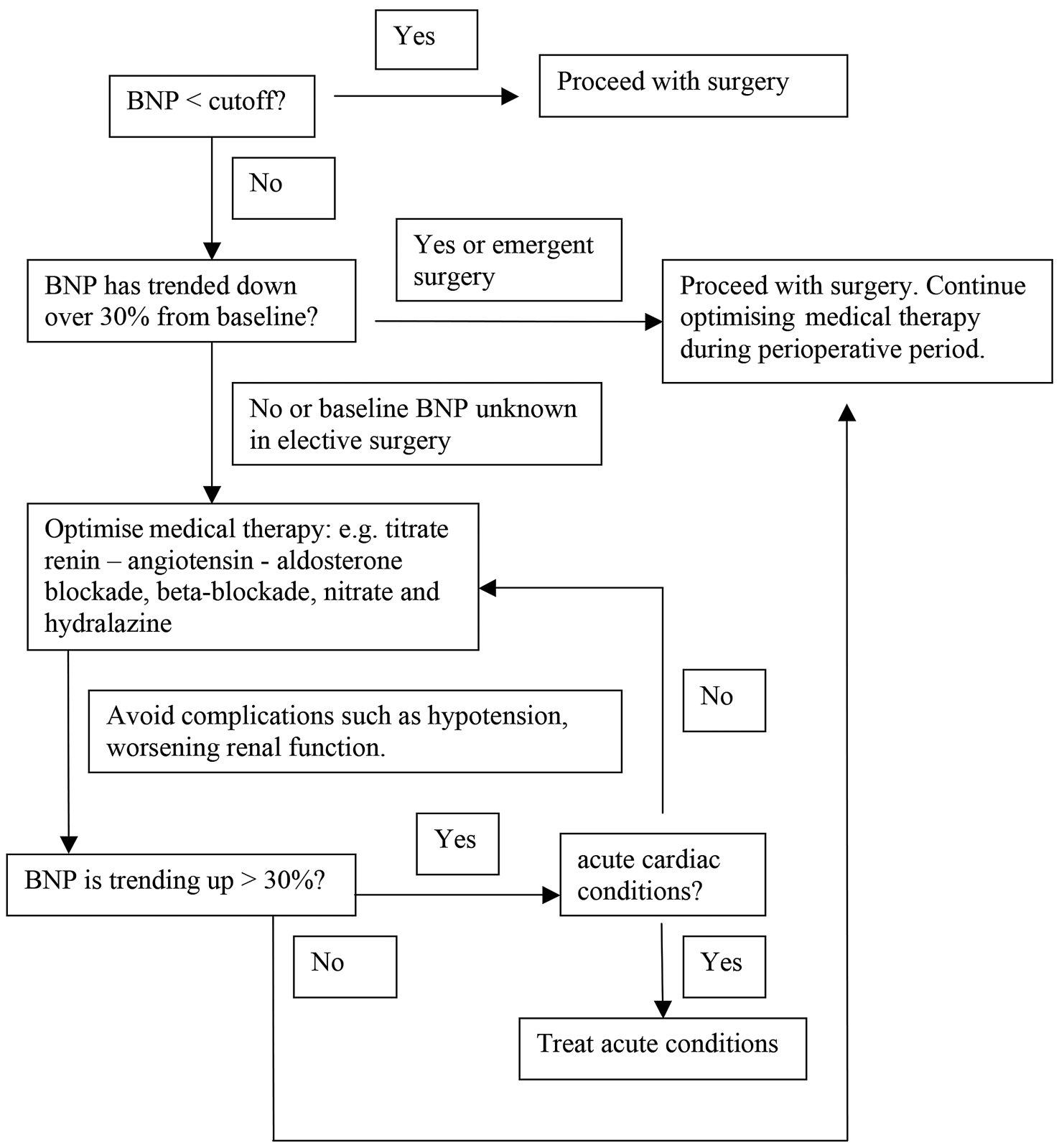

Figure 1 A proposed B-type natriuretic peptide (including amino terminal-proBNP)-guided therapy for perioperative medicine.

hypotension ${ }^{48}$ and kidney injury, nitrate may be preferred over angiotensin blockade and diuresis for patients at high risk of complications. Digoxin and cardiac resynchronisation therapy may be considered if indications are met. ${ }^{47}$ Repeat BNP testing, electrolytes and medication adjustment after five half-lives of the newly adjusted medications or sooner-depending on the stability of the patients and urgency of the surgery. If the target BNP has not been reached, medication may be adjusted during the perioperative period as long as blood pressure, renal function and electrolytes are stable. If the repeat BNP level is decreasing or fluctuating within $30 \%$ of its baseline, proceed with surgery and continue BNP-guided therapy. If BNP is trending up more than $30 \%$, this signifies high risk of cardiovascular complications $^{49}$ which requires more work-up and treatment adjustment before elective surgery. If BNP is trending up and does not respond to medication adjustment, consider evaluation of acute coronary syndrome, pulmonary embolism and other acute conditions associated with BNP increment.

\section{Incorporation of BNP testing into the current perioperative cardiac risk management algorithm}

While there is convincing evidence that BNP predicts perioperative cardiac risk, there is lack of direct evidence whether optimising perioperative BNP levels improves surgical outcome. To incorporate the ESC/ESA guideline, the following approach of incorporation of BNP test may be considered as an optional modification of current ACC/AHA algorithm for perioperative cardiac risk management while awaiting further research (figure 2): 


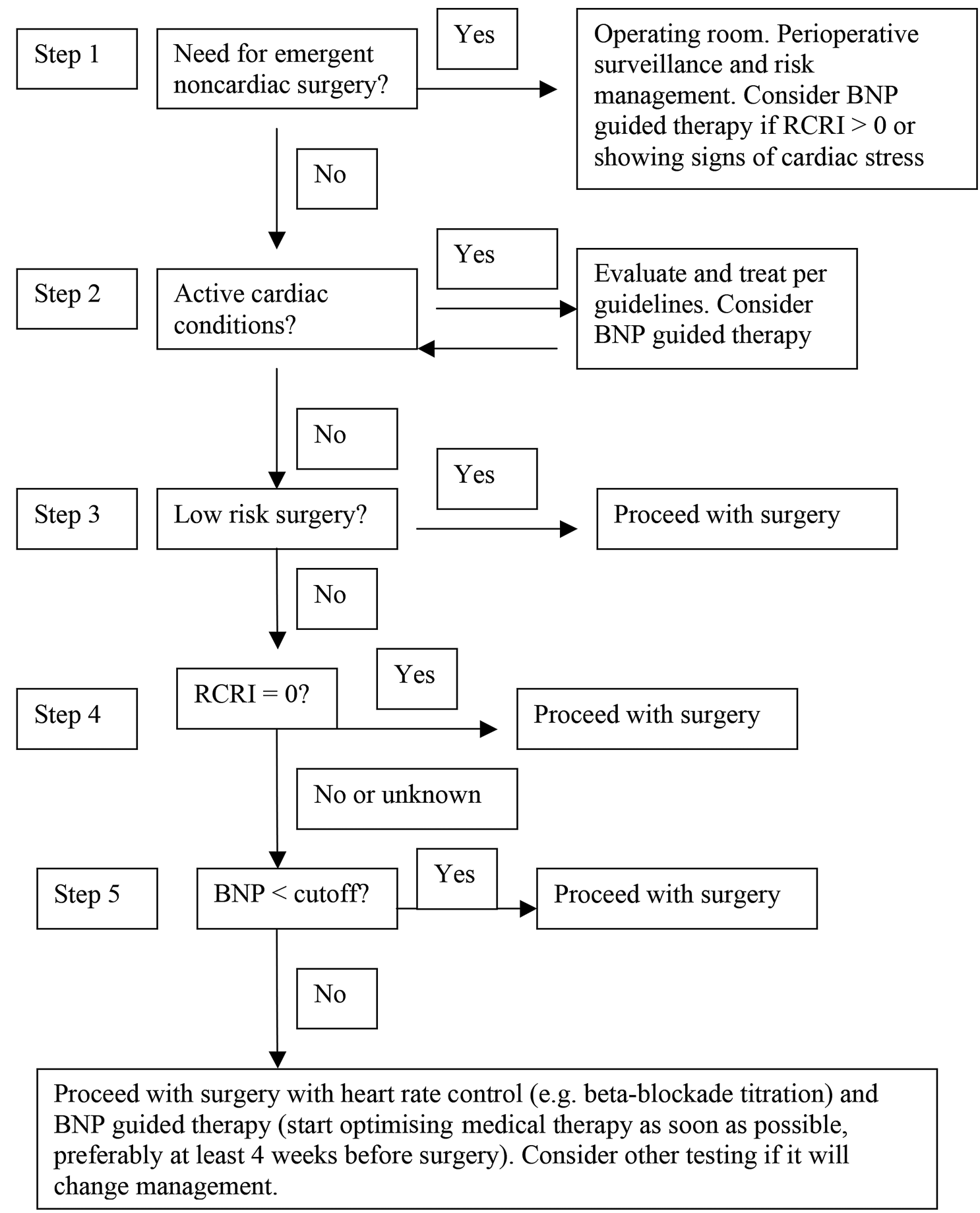

Figure 2 Cardiac evaluation and care algorithm for non-cardiac surgery-combined with B-type natriuretic peptide (BNP; including amino terminal-proBNP)-guided therapy. Modified from ACC/AHA Cardiac Evaluation and Care Algorithm for Noncardiac Surgery.

At step 1 of the modified algorithm: For emergent intermediate or high-risk surgery, consider BNP testing if RCRI $>0$ or patient meets the criteria of perioperative ECG. Consider incorporating BNP-guided therapy in perioperative surveillance and risk management if BNP is above the cut-off level.

At step 2 of the modified algorithm: Consider incorporating BNP-guided therapy in managing active cardiac conditions if the patient's BNP is above the cut-off level.
At step 3: Since the average mortality rate and hospitalisation rate for low-risk surgery are already less than $0.5 \%$ and less than $1 \%$, respectively, ${ }^{50}$ further improvement using BNP-guided therapy is unlikely to be cost-effective.

At step 4 of the algorithm: For a stable low-risk patient with RCRI of 0 , additional testing is unlikely to be cost-effective. Patient may proceed with surgery which is consistent with current guideline. ${ }^{24}$ If RCRI is more than 0, consider BNP testing. 
At step 5 of the modified algorithm, if a patient is undergoing a high-risk or intermediate-risk surgery and the patient's BNP is known to be below optimal cut-off level, patient may proceed to surgery without further testing. $^{74}$ Otherwise, consider BNP-guided therapy combined with heart rate control (mostly through $\beta$-blockade titration). For predicting perioperative cardiac risk, the OR of functional capacity assessment (walking 4 blocks or climbing 2 flights of stairs) ${ }^{25}$ is less than 2 while the OR of BNP testing is above $17 . .^{5}$ Recent systematic reviews have shown that BNP is better than functional capacity based New York Heart Association Classification in risk stratification in asymptomatic patients regardless of heart failure diagnosis. ${ }^{413}$ It is therefore a reasonable option to replace functional capacity assessment with BNP testing at this step for risk stratification. Functional capacity assessment can still be useful in preoperative evaluation as an optional addition to RCRI in risk factor stratification, that is, a combination of Fleisher-Eagle criteria ${ }^{51}$ and RCRI.

\section{BNP-guided therapy and titration of perioperative $\beta$-blockade}

BNP-guided therapy is likely to be useful in optimising $\beta$-blockade to reduce complications from $\beta$-blockade titration. Elevated BNP is associated with higher risk of mortality $^{212252}$ and stroke. ${ }^{9125354}$ BNP increase from $\beta$-blockade titration can be detected early and corrected by decreasing $\beta$-blockade or increasing renin-angiotensin-aldosterone blockade and/or nitrate + /-hydrazaline to avoid complications such as decompensation of heart failure, stroke and death shown in the POISE trial. ${ }^{55}$ The prognostic power of BNP in perioperative medicine also suggests that certain $\beta$-blockers may be preferred as they are associated with greater BNP reduction. For example, switching atenolol to carvedilol or bisoprolol has been associated with BNP reduction. ${ }^{46}$

\section{Conclusion}

BNP-guided therapy has been shown to be a safe and cost-effective treatment in heart failure though controversies still exist regarding its application in elderly with age more than 75 and in patients with severe renal impairment. It is likely a beneficial addition to perioperative medicine though the prospective RCTs in perioperative setting are still lacking. Its combination with $\beta$-blocker titration, RCRI and perioperative cardiovascular monitoring is likely to improve the outcome of surgical patients with moderate to high-cardiac risk. Since revascularisation in stable preoperative patients has been largely ineffective, BNP-guided therapy hold the promise of a more effective perioperative cardiac risk reduction, especially in a potentially synergistic combination with $\beta$-blockade titration with individualised dynamic optimisation process due to the short half-life and strong prediction power of BNP. The algorithm detailed in this article is a reasonable option to implement the current ESC/ESA guideline regarding the use of perioperative BNP testing based on current evidence.
Further research with RCTs is needed to verify the benefit of BNP-guided therapy in perioperative medicine. Based on the trials on perioperative $\beta$ blockade ${ }^{24}$ as well as the trials on BNP-guided therapy in heart failure $^{47}$ and in prevention of heart failure, ${ }^{44}$ a large multicenter trial involving thousands of patients will be needed to compare the addition of BNP-guided therapy versus current standard care in perioperative medicine. Based on the current trend of research results, ${ }^{2} 222941444656$ it is likely that future guideline on BNP testing will be similar to the current guidelines on low-density lipoprotein and blood pressure for cardiovascular risk reduction. Then the perioperative BNP testing will be part of the continuum of BNP-guided cardiovascular risk reduction which may be best started in a primary care setting as performed in the STOP-HF trial $^{44}$ long before a surgery. In multiple large cohort studies, NT-proBNP showed higher predictive value of cardiovascular events ${ }^{310}$ and mortality ${ }^{28}$ than BNP in asymptomatic patients without initial diagnosis of heart failure. The superiority of NT-proBNP is likely due to its longer half-life, therefore more consistent intraday plasma levels ${ }^{28}$ which are more reflective of baseline and less prone to transient fluctuations than those of BNP.

Acknowledgements The author thanks Sunita Patterson for editing the article, Deidre L Tomkins for reformatting the figures, Drs Melinda Gu and David Hyman for comment.

Funding This research received no specific grant from any funding agency in the public, commercial or not-for-profit sectors.

\section{Competing interests None.}

Provenance and peer review Not commissioned; externally peer reviewed.

Data sharing statement No additional data are available.

Open Access This is an Open Access article distributed in accordance with the Creative Commons Attribution Non Commercial (CC BY-NC 3.0) license, which permits others to distribute, remix, adapt, build upon this work noncommercially, and license their derivative works on different terms, provided the original work is properly cited and the use is non-commercial. See: http:// creativecommons.org/licenses/by-nc/3.0/

\section{REFERENCES}

1. Poldermans D, Bax JJ, Boersma E, et al. Guidelines for pre-operative cardiac risk assessment and perioperative cardiac management in non-cardiac surgery: the Task Force for Preoperative Cardiac Risk Assessment and Perioperative Cardiac Management in Non-cardiac Surgery of the European Society of Cardiology (ESC) and endorsed by the European Society of Anaesthesiology (ESA). Eur Heart J 2009;30:2769-812.

2. O'Donoghue M, Braunwald E. Natriuretic peptides in heart failure: should therapy be guided by BNP levels? Nat Rev Cardiol 2010;7:13-20.

3. Blankenberg S, Zeller T, Saarela O, et al. Contribution of 30 biomarkers to 10-year cardiovascular risk estimation in 2 population cohorts: the MONICA, risk, genetics, archiving, and monograph (MORGAM) biomarker project. Circulation 2010;121:2388-97.

4. Wang TJ, Gona P, Larson MG, et al. Multiple biomarkers for the prediction of first major cardiovascular events and death. $N$ Engl $J$ Med 2006;355:2631-9.

5. Karthikeyan G, Moncur RA, Levine O, et al. Is a pre-operative brain natriuretic peptide or N-terminal pro-B-type natriuretic peptide measurement an independent predictor of adverse cardiovascular outcomes within 30 days of noncardiac surgery? A systematic review and meta-analysis of observational studies. J Am Coll Cardiol 2009;54:1599-606. 
6. Ryding AD, Kumar S, Worthington AM, et al. Prognostic value of brain natriuretic peptide in noncardiac surgery: a meta-analysis. Anesthesiology 2009;111:311-19.

7. Rodseth RN, Padayachee L, Biccard BM. A meta-analysis of the utility of pre-operative brain natriuretic peptide in predicting early and intermediate-term mortality and major adverse cardiac events in vascular surgical patients. Anaesthesia 2008;63:1226-33.

8. Biccard BM, Naidoo P, De Vasconcellos K. What is the best pre-operative risk stratification tool for major adverse cardiac events following elective vascular surgery? A prospective observational cohort study evaluating pre-operative myocardial ischaemia monitoring and biomarker analysis. Anaesthesia 2012;67:389-95.

9. Shimizu Y, Nishinaga M, Takata J, et al. B-type natriuretic peptide is predictive of hospitalization in community-dwelling elderly without heart diseases. Geriatr Gerontol Int 2009;9:148-54.

10. Wang TJ, Larson MG, Levy D, et al. Plasma natriuretic peptide levels and the risk of cardiovascular events and death. $N$ Engl J Med 2004;350:655-63.

11. Witham MD, Gillespie ND, Hutcheon SD, et al. B-type natriuretic peptide is associated with mortality in older functionally impaired patients. J Am Geriatr Soc 2005;53:1991-5.

12. Omland T, Sabatine MS, Jablonski KA, et al. Prognostic value of B-Type natriuretic peptides in patients with stable coronary artery disease: the PEACE Trial. J Am Coll Cardiol 2007;50:205-14.

13. Doust JA, Pietrzak E, Dobson A, et al. How well does B-type natriuretic peptide predict death and cardiac events in patients with heart failure: systematic review. BMJ 2005;330:625.

14. Mark DB, Felker GM. B-type natriuretic peptide-a biomarker for all seasons? N Engl J Med 2004;350:718-20.

15. Gibson SC, Payne CJ, Byrne DS, et al. B-type natriuretic peptide predicts cardiac morbidity and mortality after major surgery. $\mathrm{Br} \mathrm{J}$ Surg 2007;94:903-9.

16. Ford MK, Beattie WS, Wijeysundera DN. Systematic review: prediction of perioperative cardiac complications and mortality by the revised cardiac risk index. Ann Intern Med 2010; 152:26-35.

17. Yun $\mathrm{KH}$, Jeong $\mathrm{MH}$, Oh SK, et al. Preoperative plasma N-terminal pro-brain natriuretic peptide concentration and perioperative cardiovascular risk in elderly patients. Circ $J$ 2008;72:195-9.

18. Choi JH, Cho DK, Song YB, et al. Preoperative NT-proBNP and CRP predict perioperative major cardiovascular events in non-cardiac surgery. Heart 2010;96:56-62.

19. Bolliger D, Seeberger MD, Lurati Buse GA, et al. A preliminary report on the prognostic significance of preoperative brain natriuretic peptide and postoperative cardiac troponin in patients undergoing major vascular surgery. Anesth Analg 2009;108:1069-75.

20. Cuthbertson BH, Amiri AR, Croal BL, et al. Utility of B-type natriuretic peptide in predicting perioperative cardiac events in patients undergoing major non-cardiac surgery. Br J Anaesth 2007;99:170-6.

21. Kistorp C, Raymond I, Pedersen F, et al. N-terminal pro-brain natriuretic peptide, $\mathrm{C}$-reactive protein, and urinary albumin levels as predictors of mortality and cardiovascular events in older adults. JAMA 2005;293:1609-16.

22. McKie PM, Cataliotti A, Lahr BD, et al. The prognostic value of $\mathrm{N}$-terminal pro-B-type natriuretic peptide for death and cardiovascular events in healthy normal and stage $A / B$ heart failure subjects. J Am Coll Cardiol 2010;55:2140-7.

23. Daniels LB. Natriuretic peptides and assessment of cardiovascular disease risk in asymptomatic persons. Curr Cardiovasc Risk Rep 2010;4:120-7

24. Fleisher LA, Beckman JA, Brown KA, et al.; American College of Cardiology Foundation/American Heart Association Task Force on Practice Guidelines, American Society of Echocardiography, American Society of Nuclear Cardiology, Heart Rhythm Society, Society of Cardiovascular Anesthesiologists, Society for Cardiovascular Angiography and Interventions 2009 ACCF/AHA focused update on perioperative beta blockade incorporated into the ACC/AHA 2007 guidelines on perioperative cardiovascular evaluation and care for noncardiac surgery. J Am Coll Cardiol 2009;54:e13-18

25. Reilly DF, McNeely MJ, Doerner D, et al. Self-reported exercise tolerance and the risk of serious perioperative complications. Arch Intern Med 1999;159:2185-92.

26. Hutcheon SD, Gillespie ND, Struthers AD, et al. B-type natriuretic peptide in the diagnosis of cardiac disease in elderly day hospital patients. Age Ageing 2002;31:295-301.

27. Rodseth RN, Lurati Buse GA, Bolliger D, et al. The predictive ability of pre-operative B-type natriuretic peptide in vascular patients for major adverse cardiac events: an individual patient data meta-analysis. J Am Coll Cardiol 2011;58:522-9.

28. McKie PM, Rodeheffer RJ, Cataliotti A, et al. Amino-terminal pro-B-type natriuretic peptide and B-type natriuretic peptide: biomarkers for mortality in a large community-based cohort free of heart failure. Hypertension 2006;47:874-80.

29. deFilippi CR, Christenson RH, Gottdiener JS, et al. Dynamic cardiovascular risk assessment in elderly people. The role of repeated N-terminal pro-B-type natriuretic peptide testing. J Am Coll Cardiol 2010;55:441-50.

30. Maisel A, Mueller C, Adams K Jr, et al. State of the art: using natriuretic peptide levels in clinical practice. Eur $\mathrm{J}$ Heart Fail 2008;10:824-39.

31. Tepper D, Harris S, Ip R. The role of $\mathrm{N}$-terminal pro-brain natriuretic peptide and echocardiography for screening asymptomatic left ventricular dysfunction in a population at high risk for heart failure: the PROBE-HF study. Congest Heart Fail 2009;15:296.

32. Betti I, Castelli G, Barchielli A, et al. The role of N-terminal PRO-brain natriuretic peptide and echocardiography for screening ssymptomatic left ventricular dysfunction in a population at high risk for heart failure. The PROBE-HF study. J Card Fail 2009;15:377-84.

33. Noman A, George J, Struthers A. A new use for B-type natriuretic peptide: to detect myocardial ischaemia in non-heart failure patients. Br J Diabetes Vasc Dis 2010;10:78-82.

34. Galasko GIW, Lahiri A, Barnes SC, et al. What is the normal range for $\mathrm{N}$-terminal pro-brain natriuretic peptide? How well does this normal range screen for cardiovascular disease? Eur Heart $J$ 2005;26:2269-76.

35. Goei D, Schouten O, Boersma E, et al. Influence of renal function on the usefulness of N-terminal pro-B-type natriuretic peptide as a prognostic cardiac risk marker in patients undergoing noncardiac vascular surgery. Am J Cardiol 2008;101:122-6.

36. DeFilippi C, van Kimmenade RRJ, Pinto YM. Amino-terminal proB-type natriuretic peptide testing in renal disease. Am J Cardiol 2008;101(3, Supplement 1):S82-8.

37. Madsen LH, Ladefoged S, Corell P, et al. N-terminal pro brain natriuretic peptide predicts mortality in patients with end-stage renal disease in hemodialysis. Kidney Int 2007;71:548-54.

38. Garcia S, Moritz TE, Goldman S, et al. Perioperative complications after vascular surgery are predicted by the revised cardiac risk index but are not reduced in high-risk subsets with preoperative revascularization. Circ Cardiovasc Qual Outcomes 2009;2:73-7.

39. Felker GM, Hasselblad V, Hernandez AF, et al. Biomarker-guided therapy in chronic heart failure: a meta-analysis of randomized controlled trials. Am Heart J 2009; 158:422-30.

40. Morimoto T, Hayashino Y, Shimbo T, et al. Is B-type natriuretic peptide-guided heart failure management cost-effective? Int $J$ Cardiol 2004;96:177-81.

41. Heidenreich PA, Gubens MA, Fonarow GC, et al. Cost-effectiveness of screening with B-type natriuretic peptide to identify patients with reduced left ventricular ejection fraction. J Am Coll Cardiol 2004;43:1019-26.

42. Bolliger D, Seeberger MD, Filipovic M. Pre-operative cardiac risk sssessment in noncardiac surgery: are natriuretic peptides the magic bullet? J Am Coll Cardiol 2009;54(17):1607-8.

43. Porapakkham $\mathrm{P}$, Porapakkham $\mathrm{P}$, Zimmet $\mathrm{H}$, et al. B-type natriuretic peptide-guided heart failure therapy: a meta-analysis. Arch Intern Med 2010;170:507-14

44. Ledwidge M, Gallagher J, Conlon C, et al. Natriuretic peptide-based screening and collaborative care for heart failure: the STOP-HF randomized trial. JAMA 2013;310:66-74.

45. Januzzi JL Jr, Rehman SU, Mohammed AA, et al. Use of amino-terminal proB-type natriuretic peptide to guide outpatient therapy of patients with chronic left ventricular systolic dysfunction. $J$ Am Coll Cardiol 2011:58:1881-9.

46. Mant D, Hobbs FR, Glasziou P, et al. Identification and guided treatment of ventricular dysfunction in general practice using blood B-type natriuretic peptide. Br J Gen Pract 2008;58:393-9.

47. Maisel A. Natriuretic peptide-guided therapy for heart failure: ready for "battle" or too "scarred" by the challenges of trial design? J Am Coll Cardiol 2009;55:61-4.

48. Kheterpal S, Khodaparast O, Shanks A, et al. Chronic angiotensinconverting enzyme inhibitor or angiotensin receptor blocker therapy combined with diuretic therapy is associated with increased episodes of hypotension in noncardiac surgery. $J$ Cardiothorac Vasc Anesth 2008:22:180-6.

49. Bettencourt P, Azevedo A, Pimenta J, et al. N-terminal-pro-brain natriuretic peptide predicts outcome after hospital discharge in heart failure patients. Circulation 2004;110:2168-74. 
50. Fleisher LA, Pasternak LR, Herbert R, et al. Inpatient hospital admission and death after outpatient surgery in elderly patients: importance of patient and system characteristics and location of care. Arch Surg 2004;139:67-72.

51. Fleisher LA, Eagle KA. Lowering cardiac risk in noncardiac surgery. New Engl J Med 2001;345:1677-82.

52. Berger R, Huelsman M, Strecker K, et al. B-type natriuretic peptide predicts sudden death in patients with chronic heart failure. Circulation 2002:105:2392-7.

53. Winkler K, Wanner C, Drechsler C, et al. Change in $\mathrm{N}$-terminal-pro-B-type-natriuretic-peptide and the risk of sudden death, stroke, myocardial infarction, and all-cause mortality in diabetic dialysis patients. Eur Heart J 2008;29:2092-9.

54. Reinhardt $\mathrm{F}$, Altmann $\mathrm{O}$, Reichmann $\mathrm{H}$, et al. BNP- and troponin I level in different etiology and topography of stroke. Akt Neurol 2012;39:168-73.

55. Devereaux PJ, Yang H, Yusuf S, et al.; POISE Study Group. Effects of extended-release metoprolol succinate in patients undergoing non-cardiac surgery (POISE trial): a randomised controlled trial. Lancet 2008;371:1839-47.

56. Cowie MR, Collinson PO, Dargie $\mathrm{H}$, et al. Recommendations on the clinical use of B-type natriuretic peptide testing (BNP or NTproBNP) in the UK and Ireland. Br J Cardiol 2010;17:76-80. 